\title{
A NOTE ON SIMPLE DUALITY
}

\author{
JAMES F. DAVIS ${ }^{1}$ AND PETER LÖFFLER
}

\begin{abstract}
Certain spaces satisfying Poincaré duality are shown to have the homotopy type of simple Poincare complexes. The motivation for and applications of this result are the study of free actions of finite groups on manifolds.
\end{abstract}

Let $X$ be a finite CW complex with finite fundamental group $\pi$. Then $X$ is an $n$-dimensional Poincaré complex if there is a class $[X] \in H_{n}(X)$ such that the slant product with the transfer of $[X]$,

$$
\bigcap[X]: C^{n-*}(\tilde{X}) \rightarrow C_{*}(\tilde{X}),
$$

induces a chain homotopy equivalence. Let $H$ be a quotient of

$$
\mathrm{Wh}(\pi)=K_{1}(\mathbf{Z} \pi) /\langle \pm \pi\rangle \text {. }
$$

Then $X$ is an $H$-simple Poincaré complex if the torsion $\operatorname{im}(\tau(\bigcap[X]))=0 \in H$. For example:

(i) Wh $(\pi)$-simple Poincaré complexes are called simple. Any closed manifold is a simple Poincaré complex.

(ii) $\mathrm{Wh}^{\prime}(\pi)$-simple Poincaré complexes are weakly-simple, where $\mathrm{Wh}^{\prime}(\pi)=$ $\operatorname{im}\left(K_{1}(\mathbf{Z} \pi) \rightarrow K_{1}(\mathbf{Q} \pi)\right) /\langle \pm \pi\rangle$. C. T. C. Wall [7] proved that every odd-dimensional $X$ is weakly-simple.

(iii) H. Bass [1] showed that $\mathrm{Wh}(\pi)=\mathrm{Wh}^{\prime}(\pi)$ for $\pi$ cyclic. Thus every odddimensional Poincaré complex with cyclic fundamental group is simple.

1. Simple duality. Let $A=\operatorname{im}\left(K_{1}(\mathbf{Z} \pi) \rightarrow K_{1}\left(\mathbf{Z}_{(\pi)} \pi\right)\right) /\langle \pm \pi\rangle$. Here $\mathbf{Z}_{(\pi)}$ means we invert all primes which do not divide the order of $\pi$. The object of this section is to prove

THEOREM 1.1. Let $f: X \rightarrow Y$ be a map between $n$-dimensional Poincaré complexes with $n$ even, inducing isomorphisms

$$
f_{*}: \pi_{1} X \rightarrow \pi_{1} Y, \quad f_{*}: H_{*}\left(\tilde{X} ; \mathbf{Z}_{(\pi)}\right) \rightarrow H_{*}\left(\tilde{Y} ; \mathbf{Z}_{(\pi)}\right),
$$

where $\pi=\pi_{1} X$ is finite. Assume that the induced action of $\pi$ on $H_{*}(\tilde{X} ; \mathbf{Z}[1 /|\pi|])$ and $H_{*}(\tilde{Y} ; \mathbf{Z} /[1 /|\pi|])$ is trivial. Then $X$ has the homotopy type of an A-simple Poincaré complex if and only if $Y$ does.

REMARK. In particular the theorem is true if $A$-simple is replaced by weaklysimple. If the 2-torsion of Oliver's group $\mathrm{Cl}_{1}(\mathbf{Z} \pi)$ vanishes, then $K_{1}(\mathbf{Z} \pi) \rightarrow A$ is

Received by the editors May 30, 1984 and, in revised form, August 6, 1984.

1980 Mathematics Subject Classification. Primary 57P10; Secondary 57Q10, 57Q12, 57 S17.

Key words and phrases. Simple duality, Reidemeister torsion.

${ }^{1}$ Partially supported by an NSF grant. 
an isomorphism modulo odd torsion. In this case any $A$-simple Poincaré complex has the homotopy type of a simple Poincaré complex. If the 2-Sylow subgroup of $\pi$ is cyclic, the 2 -torsion of $\mathrm{Cl}_{1}(\mathbf{Z} \pi)$ vanishes by [5]. Thus we have

COROLlaRY 1.2. Let $f: X \rightarrow Y$ as above. If the 2-Sylow subgroup of $\pi$ is cyclic, then $X$ has the homotopy type of a simple Poincaré complex if and only if $Y$ does.

Our major tool is the Bass localization sequence [1]. Let $T$ be a multiplicative subset of a ring $\Lambda$. By a $(\Lambda, T)$-module we mean a finitely generated $T$-torsion $\Lambda$-module of homological dimension one. Let $K_{1}(\Lambda, T)$ denote the abelian group resulting from the Grothendieck construction on the category of $(\Lambda, T)$-modules. There is an exact sequence

$$
K_{1}(\Lambda) \rightarrow K_{1}\left(T^{-1} \Lambda\right) \rightarrow K_{1}(\Lambda, T) \stackrel{\sigma}{\rightarrow} K_{0}(\Lambda) \rightarrow K_{0}\left(T^{-1} \Lambda\right) .
$$

Let $C$ be a finite dimensional chain complex of finitely generated free $\Lambda$-modules such that $H_{i}(C)$ is a $(\Lambda, T)$-module for all $i$. Then the Reidemeister torsion

$$
\Delta(C) \in K_{1}\left(T^{-1} \Lambda\right) / K_{1}(\Lambda)=\operatorname{ker}(\sigma)
$$

is defined by giving $C$ any $\Lambda$-base and computing the torsion of the based acyclic complex $T^{-1} \Lambda \otimes_{\Lambda} C$. We now quote from [3]:

Proposition 1.3. The Reidemeister torsion $\Delta(C)$ is equal to the torsion characteristic $\chi\left(H_{*}(C)\right)=\sum(-1)^{i}\left[H_{i}(C)\right] \in \operatorname{ker} \sigma$.

The following lemma applies even in the simply-connected case.

LEMMA 1.4. Let $g: V \rightarrow W$ be a map between $n$-dimensional Poincaré complexes with $n$ even which induces an isomorphism $g_{*}: H_{*}(V ; \mathbf{Q}) \rightarrow H_{*}(W ; \mathbf{Q})$. If the Euler characteristic $\chi(W)=0$, then

$$
\prod_{i \text { even }}\left|H_{i}(g)\right|=\prod_{i \text { odd }}\left|H_{i}(g)\right| \text {. }
$$

REMARK. If the degree of $g$ were one, then this would simply follow from Poincaré duality since then $H_{i}(g) \simeq H_{n-i+1}(g)$. For general degrees this does not hold, so different techniques are necessary.

ProOF OF 1.4. Applying the previous proposition with $\Lambda=\mathbf{Z}, T=\mathbf{Z}-0$, it suffices to prove $\Delta(g)=0 \in K_{1}(\mathbf{Z}, \mathbf{Z}-0)$. Equivalently we show

$$
\tau(g)=0 \in \tilde{K}_{1}(\mathbf{Q})=K_{1}(\mathbf{Q}) / K_{1}(\mathbf{Z}) \simeq K_{1}(\mathbf{Z}, \mathbf{Z}-0) .
$$

If $R$ is a ring, and $\alpha: M \rightarrow N$ is a homomorphism of Z-modules we write $\alpha_{R}$ for $\alpha \otimes \operatorname{id}_{R}$. Choose fundamental classes $[V],[W]$, and an integer $k$ such that $g_{\#}[V]=k[W] \in C_{n}(V ; \mathbf{Q})$. Consider the following commutative diagram:

$$
\begin{array}{ccc}
C^{n-*}(W ; \mathbf{Q}) & \stackrel{g_{Q}^{\#}}{\longrightarrow} & C^{n-*}(V ; \mathbf{Q}) \\
\cap k[W]_{\mathbf{Q} \downarrow} & & \cap[V f]_{\mathbf{Q} \downarrow} \\
C_{*}(W ; \mathbf{Q}) & \stackrel{g_{\# \mathbf{Q}}}{\longleftarrow} & C_{*}(V ; \mathbf{Q})
\end{array}
$$


Then

$$
\begin{aligned}
\tau\left(\bigcap k[W]_{\mathbf{Q}}\right) & =\tau\left(g_{\# \mathbf{Q}}\right)+\tau\left(g_{\mathbf{Q}}^{\#}\right)+\tau\left(\bigcap[V]_{\mathbf{Q}}\right) \\
& =\tau(g)+(-1)^{n} \tau(g)+\tau\left(\bigcap[V]_{\mathbf{Q}}\right) \\
& =2 \tau(g)+\tau\left(\bigcap[V]_{\mathbf{Q}}\right)=2 \tau(g),
\end{aligned}
$$

since $\tau\left(\bigcap[V]_{\mathbf{Q}}\right)=\operatorname{im} \tau(\bigcap[V])$ and $\tau(\bigcap[V]) \in \tilde{K}_{1}(\mathbf{Z})=0$. Utilizing the fact that the map $C \rightarrow C$ given by multiplication by $k$ has torsion $\left[k^{\chi(C)}\right]$, one can show

$$
\tau\left(\bigcap k[W]_{\mathbf{Q}}\right)=\tau\left(\bigcap[W]_{\mathbf{Q}}\right)+\left[(k)^{\chi W}\right]=0 .
$$

Thus $2 \tau(g)=0 \in \tilde{K}_{1}(\mathbf{Q})$ which is torsion free. Hence $\Delta(g)=\chi\left(H_{*}(C)\right)=0$.

LEMMA 1.5. In the situation of 1.1 , the torsion $\tau(f) \in A \subset \mathrm{Wh}\left(\mathrm{Z}_{(\pi)} \pi\right)$ for $\pi \neq 1$.

ProOF. Since the action of $\pi$ is free on $\tilde{Y}$, and trivial on $H_{*}(\tilde{Y} ; \mathbf{Q})$,

$$
|\pi| \chi(Y)=\chi(\tilde{Y})=\chi(Y)
$$

so that $\chi(\tilde{Y})=0$. Thus

$$
\Delta(f)=\operatorname{im} \tau(f) \in \mathrm{Wh}\left(\mathbf{Z}_{(\pi)} \pi\right) / \mathrm{Wh}(\pi)
$$

is equal to $\sum(-1)^{i}\left[H_{i}(\tilde{f})\right]$ (by 1.3) and hence to 0 (by 1.4 and the fact that the $\pi$ action on $H_{i}(\tilde{f})$ is trivial). To justify the application of 1.3 we remark that $H_{i}(\tilde{f})$ is torsion prime to $|\pi|$, and hence has homological dimension one over $\mathbf{Z} \pi$.

To finish up the proof of 1.1 , we let $R=\mathbf{Z}_{(\pi)}$ and consider the diagram:

$$
\begin{array}{ccc}
C^{n-*}(\tilde{Y} ; R) & \stackrel{\tilde{f}_{R}^{\#}}{\longrightarrow} & C^{n-*}(\tilde{X} ; R) \\
\cap k[Y]_{R} \downarrow & & \cap[X]_{R} \downarrow \\
C_{*}(\tilde{Y} ; R) & \stackrel{\tilde{f}_{\# R}}{\longleftarrow} & C_{*}(\tilde{X} ; R)
\end{array}
$$

Thus $\tau\left(\bigcap k[Y]_{R}\right)=\tau(f)+(-1)^{n} \overline{\tau(f)}+\tau\left(\bigcap[X]_{R}\right)$. Since $\chi(Y)=0$, we have

$$
\tau\left(\bigcap[Y]_{R}\right)=\tau(f)+(-1)^{n} \overline{\tau(f)}+\tau\left(\bigcap[X]_{R}\right) \in \mathrm{Wh}\left(\mathbf{Z}_{(\pi)} \pi\right)
$$

Now suppose $X$ is an $A$-simple Poincaré complex. Choose a homotopy equivalence $h: Y^{\prime} \rightarrow Y$ such that $\operatorname{im} \tau(h)=\tau(f) \in A \subset \mathrm{Wh}\left(\mathbf{Z}_{(\pi)} \pi\right)$. Then our usual diagram shows that $Y$ is $A$-simple. Similarly, if $Y$ is $A$-simple, there is an $A$-simple $X^{\prime}$. This completes the proof of 1.1 .

The above ideas can be applied to a more general situation. We call finite $\mathrm{CW}$ complexes $X$ and $Y \pi$-equivalent if $\pi=\pi_{1} X=\pi_{1} Y$ and if there exists a finite complex $Z$ with maps $X \rightarrow Z \leftarrow Y$ inducing isomorphisms on $\pi_{1}$ and $H_{*}\left(; \mathbf{Z}_{(\pi)} \pi\right)$. This is an equivalence relation on the set of finite complexes with finite fundamental group. A slight modification of the above proof gives

COROLlaRY 1.6. Let $X$ and $Y$ be $\pi$-equivalent even-dimensional Poincaré complexes with $\pi$ finite. Assume that the action of $\pi$ on $H_{*}(\tilde{X} ; \mathbf{Z}[1 /|\pi|])$ and $H_{*}(\tilde{Y} ; \mathbf{Z}[1 /|\pi|])$ is trivial. Then $X$ has the homotopy type of an $A$-simple Poincaré 
complex if and only if $Y$ does. If the 2-Sylow subgroup of $\pi$ is cyclic, then $X$ has the homotopy type of a simple Poincaré complex if and only if $Y$ does.

REMARK. If $X$ is an $n$-dimensional Poincaré complex, the obstruction to $X$ having the homotopy type of a simple Poincaré complex is an element of the Tate cohomology group $\hat{H}^{n}(\mathbf{Z} / 2 ; \mathrm{Wh}(\pi))$. Thus in our theorem $A$-simple could be replaced by simple whenever $\hat{H}^{0}(\mathbf{Z} / 2 ; \mathrm{Wh}(\pi))$ injects to $\hat{H}^{0}(\mathbf{Z} / 2 ; A)$.

REMARK. An alternative proof of 1.1 can be given, based on the fact that the relative group $K_{1}(\mathrm{Z} \pi, S)$ is torsion free, where $S$ is the set of primes to prime $|\pi|$.

2. Applications. A philosophy in transformation groups is that the geometry of a group action is present at the order of the group. This has been a motivation for the papers of Cappell and Weinberger [2] and Löffler and Raussen [4]. After summarizing some of their techniques we will show how our theorem applies to their situation. In particular we show that certain finiteness obstructions and "simplicity" obstructions vanish.

Let $\pi$ be a group of order $q$. By an ht-free $\pi$-action on $X$ we mean a free $\pi$-action where $\pi$ acts trivially on $H_{*}(X ; \mathbf{Z}[1 / q])$. Let $g: M^{n} \rightarrow N^{n}$ be a map between simply-connected closed manifolds which is a $\mathbf{Z}_{(\pi)}$-homology equivalence. If there is an ht-free $\pi$-action on $N$, there is a map $f: X / \pi \rightarrow N / \pi$ and a homotopy equivalence $h: M \rightarrow X$ such that $\tilde{f} \circ h=g$. Furthermore the $\pi$-action on $X$ is ht-free and $X / \pi$ is a Poincaré complex. Now suppose $\pi$ is a cyclic group. Surgery theoretic techniques then show that for $n>3, X / \pi$ has the homotopy type of a closed manifold if and only if $X / \pi$ has the homotopy type of a simple Poincaré complex (see [2]). (The surgery theoretic techniques involve the fact that $L_{*}^{s}(\mathbf{Z} \pi)$ is detected by multisignature, Arf invariants, and codimension one Arf invariants.) From 1.2 and (iii) we conclude

COROLlaRY 2.1. If $g: M^{n} \rightarrow N^{n}(n>3)$ is a $\mathbf{Z}_{(\pi)}$-homology equivalence between simply-connected closed manifolds and if there is an ht-free $\pi$-action on $N$, then there is an ht-free $\pi$-action on a closed manifold $M^{\prime}$, which has the homotopy type of $M$. Similarly, if there is an ht-free $\pi$-action on $M$, there is one on $N^{\prime} \simeq N$.

REMARKS. In [2] this result was proved in the following cases: $q=p^{r}$ with $p$ an odd prime, degree $g=1$, or $n$ odd. In [3] the question of what happens in odd dimensions for noncyclic groups was discussed.

Combining the above corollary with a transfer argument in the surgery exact sequence (see [2]) we deduce

COROLlARY 2.2. Let $g: M^{n} \rightarrow N^{n}(n>3)$ be a $\mathbf{Z}_{(\pi)}$-homology equivalence between simply-connected closed manifolds. If there is an ht-free $\pi$-action on $N$ such that the local normal invariant $\nu(g)$ is in the image of the transfer

$$
\left[N / \pi, G / \operatorname{TOP}_{(\pi)}\right] \rightarrow\left[N, G / \operatorname{TOP}_{(\pi)}\right]
$$

then there is an ht-free action on $M$ making $g$ equivariant.

This answers a question of S. Weinberger (see [6]) posed at the 1983 American Mathematical Society Summer Conference on Group Actions.

These corollaries are also valid when the 2-Sylow subgroup of $\pi$ is normal cyclic, provided that the finiteness obstruction vanishes:

$$
\sum(-1)^{i} \sigma\left[H_{i}(g)\right]=0 \in \tilde{K}_{0}(\mathbf{Z} \pi) .
$$


As a consequence of Lemma 1.4, this is true whenever $n$ is even.

\section{REFERENCES}

1. H. Bass, Algebraic K-theory, Benjamin, EImsford, N.Y., 1968.

2. S. Cappell and S. Weinberger, Homology propagation of group actions (to appear).

3. J. F. Davis and S. Weinberger, Group actions on homology spheres (to appear).

4. P. Löffler and M. Raussen, Symmetrien von Mannigfaltigkeiten und rationale Homotopietheorie (to appear).

5. R. Oliver, $S K_{1}$ for finite group rings. I, Invent. Math. 57 (1980), 183-204.

6. R. Schultz, Proc. 1983 Amer. Math. Soc. Summer Research Conf. on Group Actions on Manifolds (R. Schultz, ed.), Contemporary Math. (to appear).

7. C. T. C. Wall, Norms of units in grous rings, Proc. London Math. Soc. 29 (1974), 593-632.

Department of Mathematics, University of Notre Dame, Notre Dame, INDIANA 46556

MATHEMATISCHES INSTITUT DER UNIVERSitÄt GÖtTINGEN, BUNSENSTRASSE 3-5, 3400 GÖTTINGEN, WEST GERMANY 